\title{
Facilitating Self-reflection about Values and Self-care among Individuals with Chronic Conditions
}

\author{
Catherine Y. Lim \\ Kaiser Permanente \\ Washington Health \\ Research Institute \\ Seattle, WA, USA \\ catherine.y.lim@kp.org
}

\author{
Andrew B.L. Berry \\ Andrea L. Hartzler \\ ablberry@uw.edu \\ andreah@uw.edu \\ University of Washington \\ Seattle, WA, USA
}

\author{
Tad Hirsch \\ Northeastern University \\ Boston, MA, USA \\ tad.hirsch@northeastern. \\ edu
}

\author{
David S. Carrell \\ Zoë A. Bermet \\ James D. Ralston \\ david.s.carrell@kp.org \\ zoe.a.bermet@kp.org \\ james.d.ralston@kp.org \\ Kaiser Permanente \\ Washington Health \\ Research Institute
}

\begin{abstract}
Individuals with multiple chronic conditions (MCC) experience the overwhelming burden of treating $\mathrm{MCC}$ and frequently disagree with their providers on priorities for care. Aligning self-care with patients' values may improve healthcare for these patients. However, patients' values are not routinely discussed in clinical conversations and patients may not actively share this information with providers. In a qualitative field study, we interviewed 15 patients in their homes to investigate techniques that encourage patients to articulate values, self-care, and how they relate. Study activities facilitated self-reflection on values and self-care and produced varying responses, including: raising consciousness, evolving perspectives, identifying misalignments, and considering changes. We discuss how our findings extend prior work on supporting reflection in HCI and inform the design of tools for improving care for people with MCC.
\end{abstract}

\section{CCS CONCEPTS}

- Human-centered computing $\rightarrow$ Empirical studies in HCI; Interaction design.

\section{KEYWORDS}

Multiple chronic conditions, multimorbidity, reflection, selfcare, values, health

\section{ACM Reference Format:}

Catherine Y. Lim, Andrew B.L. Berry, Andrea L. Hartzler, Tad Hirsch, David S. Carrell, Zoë A. Bermet, and James D. Ralston.

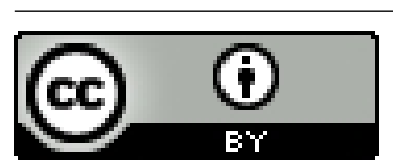

This work is licensed under a Creative Commons Attribution International 4.0 License.

(c) 2019 Copyright held by the owner/author(s).

ACM ISBN 978-1-4503-5970-2/19/05.

https://doi.org/10.1145/3290605.3300885
2019. Facilitating Self-reflection about Values and Self-care among Individuals with Chronic Conditions. In CHI Conference on $\mathrm{Hu}$ man Factors in Computing Systems Proceedings (CHI 2019), May 4-9, 2019, Glasgow, Scotland UK. ACM, New York, NY, USA, 12 pages. https://doi.org/10.1145/3290605.3300885

\section{INTRODUCTION}

People living with multiple chronic conditions (MCC) face the everyday challenges of managing illnesses amidst other demands in their daily lives [6, 7]. Often with help from family caregivers, these individuals perform the daily work of self-care, which can include: illness work (e.g., physical activity, taking medications, monitoring blood glucose levels and blood pressure), everyday life work (e.g. obligations to family, job), and biographical work (e.g., coping with the emotions around accepting and adjusting to changes in life due to illness) [16, 28]. The combination of self-care tasks for patients with MCC is often overwhelming and can be further complicated when recommendations for managing different health conditions conflict [13, 41, 45]. This can lead people with MCC to prioritize certain self-care tasks over others, often making these decisions on their own without the assistance of healthcare providers[42, 46].

Efforts to improve healthcare for the growing population of people with MCC [21,43] emphasize the need to orient care around outcomes important to patients [2, 41]. Recent work has sought to address the need to support patients, family caregivers, and healthcare providers to align care with patients' values [33]-what patients consider most important to their well-being and health [10,27]. Yet, eliciting patients' values is not a routine part of care [9]. Conversations that do occur often incorporate a limited range of values $[12,26]$. There is an opportunity for designers to help patients identify and communicate the complex relationships among their values, self-care, and other aspects of a daily life. In this study, we sought to understand how to support patients in articulating these relationships. 
We engaged 15 patients, including 6 who opted to include a family caregiver, in interviews in their homes. Using commonly available materials (e.g., index cards, paper worksheet), we experimented with a set of techniques aimed to help patients articulate connections between their values and self-care. Study activities included verbally eliciting values and self-care, externalizing these in written form, assigning ratings of importance and self-efficacy, and visually arranging values and self-care tasks to explore and articulate relationships between them. This paper makes several contributions. First, we found that reflection may be a critical first step to articulating values. This is significant because it challenges the assumption that values are inherently known. Second, we provide an account of how various techniques facilitated self-reflection and prompted a range of responses, including: raising consciousness, evolving perspectives, identifying misalignments, and considering changes to values and self-care. These findings clarify design implications for future work in improving chronic illness care and designing to support reflection in $\mathrm{HCI}$.

\section{RELATED WORK}

\section{Communication about patients' values}

To improve the quality of healthcare for individuals with MCC, the importance of orienting care around patients' values is well-documented [6, 39]. Understanding patients' values is also considered an important part of building strong patient-provider relationships [18]. Prior work on methods for eliciting and clarifying values in clinical settings typically focus on eliciting patients' preferences in making individual medical decisions from a set of closed-ended diagnostic or treatment options [44]. Recent work has sought to expand the breadth of values that may be valuable for informing individualized, patient-centered care [27, 32]. Domains of values important to people with MCC include those not typically discussed in clinical contexts: activities, possessions, principles, relationships, emotions, and abilities [10,27].

Boundaries exist that prevent patients from seeing their values as relevant to their care and actively discussing their values with their healthcare providers [26]. There is a need to address these challenges by better supporting patients to understand how their values relate to the everyday work of self-care. This is especially important for people living with MCC, who mostly manage their health at home and make many decisions daily about their priorities for self-care $[7,40,45]$.

\section{Reducing burden of treatment for MCC: a focus on values}

There is extensive prior work in HCI to support individuals who perform the daily work of managing chronic conditions
[14], such as taking medications [35] or communicating with caregivers[19, 34]. To support self-care that mostly takes place in the home, prior work has sought to integrate technologies into everyday life [1,22]. Much of this work focuses on the needs of people managing single chronic conditions. Ancker et al. found that for patients with MCC self-care technologies can be especially burdensome and create additional work [3]. To support the needs of people with MCC, there is an opportunity for designers in HCI to consider how these individuals experience a disproportionate burden of treatment, which refers to the overwhelming amount of work they face daily to manage their health [36].

To reduce the burden of treatment, clinical guidelines suggest that healthcare providers take a patient-centered approach to help patients make choices that better align selfcare with their values[36]. Beyond technologies, providers adopt existing techniques like motivational interviewing to help patients explore their motivations for performing selfcare work, so that patients can arrive at their own decisions about exploring changes to self-care [31]. Motivational interviewing is a technique intended for use by providers. There is a need to understand how such techniques may inform the design of tools for patients to use on their own or with the support of a family member or peer. Further, there is an opportunity to help people with MCC to clarify how their values relate to decisions about self-care, especially when they are overwhelmed.

\section{Designing support for reflection}

Support for reflection is a growing area of interest in HCI $[5,38]$. Recent work has sought to bring focus to reflection as an end rather than as a means to behavior change or goal attainment. Fleck and Fitzpatrick discuss the need for first clarifying the purpose of reflection, along with the intended reflective behaviors and techniques most effective for encouraging such behaviors [20]. Baumer extends this work by introducing reflective informatics, offering a theoretical concepts to inspire future work on reflection [4].

Reflection as an aspect of self-care technologies is useful for building knowledge of one's experience of disease as well as one's own behaviors and actions. Li et al. include reflection as one of five key stages for designing effective personal informatics systems, emphasizing that both short-term and long-term reflection is considered helpful for gaining self knowledge [25]. Mamykina et al.'s work on the sensemaking approach to informatics interventions for self-care ties reflection to problem-solving, emphasizing support for the process of learning from one's own experiences [29, 30]. Reflection is not only done alone. Prior work has shown the benefits of reflecting through social interactions with family members or healthcare providers [37]. 
Prior work on reflective informatics and personal health informatics help people discover patterns and insights by visualizing or displaying personal health data to draw insights [15]. Thudt et al. found that allowing people to actively construct physical representations of their data in the context of their homes induced various types of self-reflection [38]. This work focuses heavily on visualizing quantifiable personal health data, typically collected through self-tracking. There is a gap in understanding how constructing physical representations can create opportunities for self-reflection on one's values and how they relate to healthcare.

\section{METHODS}

In a qualitative field study, we conducted semi-structured interviews in participants' homes and utilized index cards to guide conversations during two-hour visits. We asked participants to complete a written worksheet before the first visit and a photo elicitation activity before the second visit. Study procedures received institutional review board approval at Kaiser Permanente Washington Health Research Institute.

\section{Participants}

We engaged 15 individuals with MCC (P1-P15), who we refer to as patients. We recruited patients from an integrated healthcare system in Washington state. To be eligible to participate, patients were required to have a diabetes diagnosis and at least two of the following common chronic conditions: depression, osteoarthritis, and coronary artery disease. We selected these conditions because they are likely to require daily self-management and treatment instructions for these conditions may conflict. We engaged participants who were living at home without the help of a professional caregiver.

Six of the patients elected for the optional inclusion of a family caregiver during interviews. All caregivers $(n=6)$ (CG1-CG6) co-habitated with the corresponding patients. Most family caregivers $(n=5)$ were spouses, and one was the child of a patient. Table 1 shows the demographics of our sample.

\section{Procedures}

We completed two home visits with each patient, each lasting approximately 1.5-2 hours. We encouraged caregivers to participate if they had anything to add, but we did not directly ask them questions.

Roughly one week before the first visit, we mailed each patient a worksheet. The worksheet included two sections. The first focused on the patients' values (e.g., "What is most important to your well-being and health?"). We provided six domains, with examples, to encourage patients to think about their values comprehensively, beyond those they considered to be medically relevant: activities, possessions, principles, relationships, emotions, and abilities [27]. The second section
Table 1: Participant Characteristics

\begin{tabular}{|c|c|c|}
\hline Characteristics & $\begin{array}{l}\text { Patient } \\
(\mathrm{n}=15)\end{array}$ & $\begin{array}{l}\text { Caregiver } \\
(\mathrm{n}=6)\end{array}$ \\
\hline \multicolumn{3}{|l|}{ Age } \\
\hline Mean $(\mathrm{SD}=8.31)$ & 71.5 & 60.2 \\
\hline Range & $58-86$ & $33-72$ \\
\hline \multicolumn{3}{|l|}{ Gender } \\
\hline Female & 9 & 2 \\
\hline Male & 6 & 4 \\
\hline \multicolumn{3}{|l|}{ Race } \\
\hline White/Caucasian & 7 & 5 \\
\hline Black or African American & 6 & 1 \\
\hline Asian & 2 & 0 \\
\hline \multicolumn{3}{|l|}{ Ethnicity } \\
\hline Hispanic/Spanish/Latino/a origin & 4 & 0 \\
\hline \multicolumn{3}{|l|}{ Education } \\
\hline Some high school & 1 & 0 \\
\hline High school degree/GED & 6 & 2 \\
\hline Some college/2-yr degree & 4 & 1 \\
\hline 4-yr college degree & 1 & 2 \\
\hline More than 4-yr college degree & 3 & 1 \\
\hline \multicolumn{3}{|l|}{ Marital Status } \\
\hline Single & 1 & 1 \\
\hline Married/partnered & 8 & 5 \\
\hline Widowed & 2 & 0 \\
\hline Divorced/separated & 3 & 0 \\
\hline No answer & 1 & 0 \\
\hline \multicolumn{3}{|l|}{ Employment } \\
\hline Employed full-time & 3 & 1 \\
\hline Employed part-time & 0 & 1 \\
\hline Retired & 10 & 4 \\
\hline Disabled/unable to work & 1 & 0 \\
\hline No answer & 1 & 0 \\
\hline \multicolumn{3}{|l|}{ Chronic conditions } \\
\hline Diabetes & 15 & $\mathrm{n} / \mathrm{a}$ \\
\hline Depression & 13 & $\mathrm{n} / \mathrm{a}$ \\
\hline Osteoarthritis & 13 & $\mathrm{n} / \mathrm{a}$ \\
\hline Coronary Artery Disease & 6 & $\mathrm{n} / \mathrm{a}$ \\
\hline
\end{tabular}

focused the patients' daily self-care activities and tasks (e.g., "What do you daily to manage your well-being and health?") and included examples to encourage patients to think comprehensively about self-care. We drew examples from prior work on types of self-management work performed by individuals with chronic conditions [16, 28]. Some examples include keeping track of conditions (e.g., measuring blood 
pressure), adjusting how daily tasks are done due to health or other changes, housekeeping or running errands, taking care of others, and managing emotions. Patients were encouraged to fill out the worksheet before the first home visit, to allow them an opportunity to think about their values and self-care in preparation for the interview.

Generating value cards. Two interviewers were present during each interview. Interviews were typically conducted around a tabletop surface, such as a dining table or coffee table. We began the first interview by asking the patient to share what they had written on the worksheet about their values. While the patient read aloud their values, an interviewer wrote each response on an index card. For patients who did not complete the worksheet in advance of the visit, we used the worksheet to guide the generation of cards. While generating cards, the interviewer arranged them on the table so each card was visible to the patient. The activity continued until the patient felt that the set of cards was a complete representation of their values.

Rating value cards. We asked patients to rate the importance of each value on a scale from 1 (important) to 5 (extremely important). We wrote the rating on the bottom left corner of each card. We then took a photo of all of the value cards laid out on the table.

Generating self-care cards. We then continued to the other section of the worksheet and created a set of index cards to represent self-care tasks. We used green cards for writing down values and white index cards for writing down selfcare tasks. We continued to generate cards until the patient felt that the set of cards was a good representation of what they do daily to manage their chronic conditions.

Rating self-care cards. Similar to the rating of value cards, we asked patients to rate the importance of a self-care card on a scale from 1 to 5 . In addition, we asked participants to rate their confidence in their ability to perform the selfcare task on each card, with a number from 1 (not at all confident) to 5 (very confident). We wrote the importance rating on the bottom left corner and the confidence rating on the bottom right corner of each card. Once the rating activity was complete, we took a photo of all of the self-care cards laid out on the table. Once we created and rated both sets of cards, we fastened each set together using key rings and left them with the patient, inviting them to add or modify the cards before the second visit.

Photo elicitation. At the end of the first visit, we asked patients to complete an optional photo elicitation activity [24] before the second visit to help them think about how values and self-care connect. We providing digital cameras and asked patients to take 1-10 photos of how items written on the index cards fit into the context of their daily lives. For each photo, patients were asked to record which cards were captured in the photo and how these items overlap or connect. We began the second home visit by asking the patient to describe each photo they took and their written responses. This method enabled patients to drive the conversation at the start of the second visit.

Articulating connections between values and self-care. During the second visit, we arranged both sets of index cards on the table so that all cards were visible. The green cards (values) were arranged in a grid on one side and the white cards (selfcare) were arranged on the opposite side. We then asked the patient to select one value card they wanted to discuss. We placed this value card in the center of the table and asked the patient to identify other cards related to the card in the center and to describe how each related. We continued with describing the connections between cards until the patient felt that they had identified all of the cards the related to the value in the center. We repeated this activity once or twice more depending on the time available.

Meta-discussion on study activities. During both visits, we prompted patients to engage in a meta-discussion about each study activity. We asked patients to describe their experience after completing each activity. We asked two feedback questions for each activity: "What, if anything was difficult about the activity you just completed?" and "What, if anything, did you learn or discover while completing this activity?"

At the end of the second home visit, we asked the patient and caregiver to complete a demographic survey. Each patient received $\$ 50$ for each home visit completed. We audio recorded the interviews and used a professional transcription service to have the recordings transcribed verbatim.

\section{Analysis}

Two authors $[\mathrm{AB}, \mathrm{CL}]$ completed the analysis, with support from all other authors. The analysis approach was informed by constructivist grounded theory methods [17]. Atlas.ti was used to collect and analyze interview transcripts. Open coding of interview transcripts began as soon as the first transcript was available. $[\mathrm{AB}, \mathrm{CL}]$ coded transcripts, wrote memos to define codes, met to discuss code definitions, refined code definitions, and iterated on this process as interviews were completed. Code refinement was achieved by comparing data to data, codes to data, and codes to codes. Once codes stabilized and a codebook finalized, [AB, CL] individually finished coding all remaining transcripts. As coding was completed, $[\mathrm{AB}, \mathrm{CL}]$ grouped codes into themes, reviewed excerpts associated with themes, and wrote theme descriptions. Refinement of themes continued until saturation was completed. As themes emerged and were refined, interview facilitators used this emerging understanding to 
influence questions and probes asked during interviews. A final set of themes are presented in the findings.

\section{FINDINGS}

We report on findings from thematic analysis of interview transcripts, which includes accounts of how participants described their values and self-care while generating cards, connections between values and self-care while arranging cards, and their feedback on study activities performed. First, we provide examples of how patients articulated connections between their values and self-care. Second, we describe, from the perspective of patients, how the techniques we utilized encouraged them to think about their values and self-care in new ways. We found that these activities encouraged selfreflection about values and self-care. Patients described a range of responses to these opportunities to reflect, including: raising consciousness, evolving perspectives, recognizing misalignments, and considering changes in their lives. Finally, we describe how some patients experienced little change to how they think about or articulate their values and self-care.

\section{Articulating relationships between values and self-care}

All patients were able to complete the activity of visually arranging the values and self-care cards to demonstrate and describe relationships among them. For example, P2 articulated relationships between the value of Thinking and being more positive in life to self-care cards of Following a routine, Playing golf, Morning exercises, and Eating a bland diet. P2 explained that being in control of his daily routine and regularly playing golf with friends who had been supportive while he received cancer treatment helped him cope with negative thoughts and, therefore, allowed him to think more positively. Like many patients, P2 shared stories to provide examples, such as negative experiences when he did not follow his morning and different ways his golfing friends were supportive to him.

We include photographs to illustrate the range of responses to the activity of arranging index cards and describing connections among values and self-care. Some patients described connections among a large number of value and self-care cards and elaborated on these connections in detail. For example, P8 (Figure 1) placed the value of Being fair, justice in the center and described connections to 10 value cards and 10 self-care cards.

In contrast, some participants articulated a smaller set of connections. P11 discussed connections to the value of Independence, taking care of myself. After placing the card in the center, P11 named three values and three self-care tasks that related to this value. Although P11 articulated which

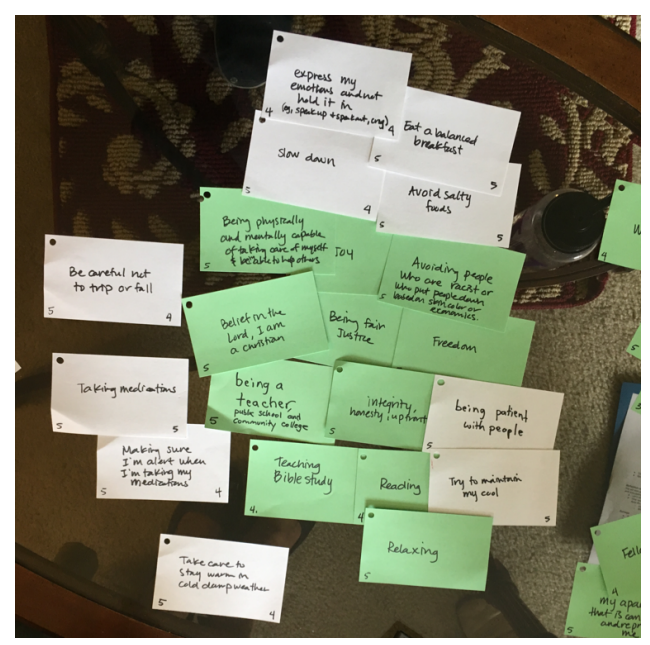

Figure 1: Arranging cards to articulate relationships between values and self-care

cards were connected, she elaborated little beyond naming the cards.

I: How is walking connected to independence?

P11: So I can walk to get somewhere.

I: What else?

P11: Having a place of my own. Being mentally sharp.

I: Yeah, how is mentally sharp connected to independence?

P11: You can't be independent unless you're mentally okay. And mobility, I guess.

Although we encouraged starting with one value card in the center, we did not impose a specific structure or spatial relationships of the cards. In fact, some patients organized the cards in columns or clusters rather than radiating from a central card. Figure 2 shows the visual arrangement of P5's cards. This arrangement began with a central value of Property in [city redacted] (the family's vacation home), which ended up at the top of the right column. These examples of how patients arranged and articulated the relationships between values and self-care demonstrate how the technique produced varying responses.

\section{Occasions to "slow down and think" about values and self-care}

We found that patients found the study activities useful as opportunities to think in new ways about their values, selfcare, and how they relate. These moments contributed to deeper understandings of their own values and chronic illness care in daily life. P9 described his experience as a process of "awakening." P8 expressed appreciation for the occasion to "slow down and think": 


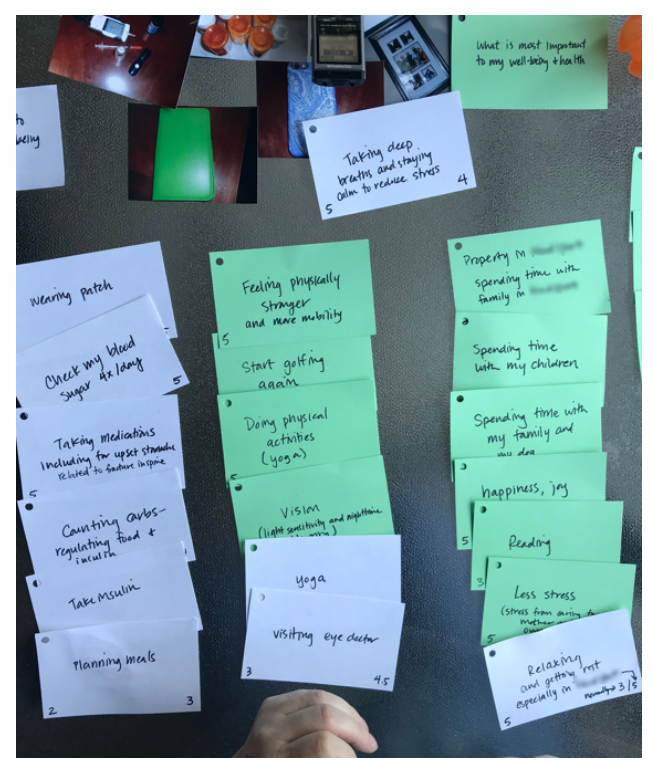

Figure 2: Second example of arranging cards relating values and self-care

"You're just busy doing this and doing that. And it slowed me down enough, that's why I say I need to slow down and think. My dad used to have a saying, "Being able to think is a powerful thing.' When you just stop and begin to think, and I haven't been doing much of that. But since I've been talking with you, especially this activity, it's been very helpful to point me in that direction more." (P8)

Patients' propensity to discuss, question, and develop deeper understandings of their values and self-care varied. Patients described how various study activities prompted them to reflect. In the next section we describe four themes that emerged from our analysis: raising consciousness, evolving perspectives, recognizing misalignments, and considering changes they might make in life. We describe how some patients experienced little to no change in how they thought about their values and self-care despite completing all study activities.

Raising consciousness. Participants described the study activities as occasions to consider aspects of their daily life in ways they do not do routinely. This self-reflection caused them to bring awareness to aspects of daily lives, including values and self-care work, that they believed to be tacitly known:

"I think it was conscious raising, a reminder and thought provoking for me to recapture some things that I put in the back of my mind or tried to shut out and come forward and think, how does this affect my well-being? Because it's been a long time that I have not focused on my wellbeing. And so it's good for me to be doing that." (P8)

Some participants realized certain values or self-care work were more important than they originally thought. They made discoveries about the significance they placed on certain values. For example, P15 found the activity of assigning importance and confidence ratings to self-care to challenge some of his assumptions. For P10, who worked as a bus driver, prompts to articulate habitual or routine tasks and self-care activities brought a consciousness to the self-care work she does daily:

"I mean, it's like when I drive my bus, I just drive my bus. I know everything I need to do. I don't think about it. I just do it. But it made me think, about a lot of different stuff I do on a daily basis that I don't think about it. I just do it. It's like people breathe and you don't think about breathing. You just breathe." (P10)

P9 took several days to complete the written worksheet provided before the first visit. He described why thinking about his values took careful thinking and added "dimensions" to how he thought about his well-being and health.:

'It's one thing to read something, but then you read it and then think about it and have to analyze it. You have to put a little more work into it, so in the beginning it was kind of challenging because you got to do a self search, because you want to be honest with yourself because you're talking about yourself. But it gave me some insight, the things that I do that I don't think about in terms of something that's right or something that's important, it's just something that's part of my life. It adds a little bit of a different dimension." (P9)

For some participants, raising consciousness of personal values and self-care routines led to an occasion to express gratitude for what they have in their daily lives. For example, looking across the index cards that represented his personal values caused $\mathrm{P} 2$ to be more aware of the richness of relationships important to him. P2 expressed gratitude for friends and family who supported him through cancer treatment and recovery:

"I discovered that friendship is something that is very important to me. And that I'm a lucky person that the people I run into are such a support to me." (P2) 
Raising consciousness of personal values, self-care, and how they relate are some common responses of participants across all study activities.

Evolving perspectives. We found that patients changed or evolved their perspectives on values and self-care. Changing definitions of values or self-care or stating changes to the rating they placed on a particular value or self-care activity were common examples. Typically these changes occurred during the second home visit, when participants were prompted to draw relationships between their personal values and self-care activities in daily life.

One example of how participants evolved their perspectives was the revision of definitions for values or self-care. When drawing connections between her personal value of Being fair, justice and other values and self-care, P8 reexamined and revised her definition of Being fair, justice. Initially, P8 described this value as pertaining to her being fair and just to other people and justice for society. P8 then readdressed the value in relation to being fair and just to herself:

"As we just concluded, there are three perspectives on this. One is being fair and just to myself, being fair and just to others, and being fair and just as it relates to issues that impact others, like civil rights and making sure that justice does prevail. So if I look at this one, take care to stay warm in cold damp weather, it's being fair to myself so I don't get sick. And making sure I'm alert and focused when I take my medications so I'm being fair to myself and I don't end up with the wrong medicine...I think the three perspectives that evolved from our discussion has been something for me to further reflect on." (P8)

When discussing the importance rating, some participants were surprised by how much importance they placed on certain values. For example, P12 initially stated that she did not place importance on physical possessions. While assigning numerical ratings to each value, P12 discovered that an old car had more significance than she had originally considered.

"I didn't realize how much this car meant to me until we got talking about. And I said oh no, I don't hold onto possessions. Oh yeah, I do." (P12)

Similarly, the activity of rating the importance of values was a different way for P7 to think. P7 described how he takes time to think during his long commute, prioritizing what is important to him. However, he had never used numerical ratings, so the study activity prompted him to think differently than he typically does. Despite being initially skeptical about the utility of numerical ratings, P7 explained that the activity was "mind-opening." Additionally, he realized that importance ratings he had assigned during the first visit may have changed by the time of the second visit, along with some changes to his housing situation. Importance and confidence ratings were more conditional than he had originally thought. He re-evaluated the importance ratings of each value while describing how they were related (or unrelated) to the value of Being aware of immediate health problems:

"Got to be honest with myself. Is it that important?...No, but it's something down the road that's going to make me happy because I like doing it. It keeps me more active. But it doesn't have anything to do with immediate health. 'Imagination'? I don't need imagination, I need answers, and I don't have the answers so I need to have somebody with the knowledge to give me the answers that I need so I can make a page for myself. 'Honesty, openness'? I've already crossed that base, I've already done that with all these things. 'Being flexible with other people'? No, I'm not going to be flexible because it's got to do with my life and my health. I can't be flexible..." (P7)

Recognizing misalignments. Patients who recognized misalignments often described discovering differences between what they do daily and what they stated as important. In some cases, this meant that patients re-examined the veracity of their original statements about personal values. P12 described a personal value of Telling it like it is, which referred to speaking with honesty and candor. She felt this was especially important in communication with her children and grandchildren and when she spoke with healthcare providers. When she described how other values and selfcare cards were related to Telling it like it is she realized that she was, in reality, more selective about Telling it like it is than she originally thought:
"It's making me realize I don't really tell it like it is because I'm leaving out certain people because I know how it'll affect certain people. Hadn't thought about it. Except, well, kids, grandkids, I've thought about that. Should I tell them [bad news about my health] or should I not? Most of the time not. So I'm not being honest with some of them." (P12)

Another example of participants recognizing misalignments arose during the rating activity, when participants found misalignments between the importance they placed on some personal values compared to the frequency with which the value occurred in daily life. For example, P10 recognized the discrepancy between the time she spent with her family 
compared to time spent on activities she thought were less important:

"If some things are not important to me, even
though I may do it more often than I do some-
thing that's really important to me. You under-
stand what I'm saying? So it's like a challenge.
Do I want to give it a high rating or do I want
to give it a low rating? Like watching movies. I
love to watch movies, but it's not that important
to me. It's important enough to me that I really
love to do it, but it's not that important to me
that I will put it above something else like my
family or something like that." (P10)

On some occasions patients' account of their values or selfcare conflicted with the family caregiver's account of the patient's daily life. P3 was especially troubled with whether Watching TV belonged as a personal value. His son, CG3, chimed in and pointed out that it must be an important activity because he observed P3 sitting in front of the TV for many hours each day:

P3: Now that's the only reason, the TV. What's most important to your well-being and health, you could say 'not watching TV'. No...Leave it as it is...

CG3: If he didn't find some value in it, he wouldn't be sitting in front of the TV for eight hours a day. Good or bad, there's something there.

Patients recognized several types of misalignments: between their statements about values and the veracity of statements, between importance or confidence ratings and the actual frequency of a self-care activity was performed, and between patients' descriptions and family caregivers' descriptions of personal values and self-care.

Considering changes. We found that participants considered making changes in their daily lives after engaging in study activities. These included changes in self-care or major changes in life, such as whether to retire. P8 described how she was considering changes she could make to increase physical activity and relaxation. This occurred after a discussion about a misalignment between the importance and confidence ratings she assigned to the self-care card of Increasing physical activity:

"I need to change my daily routine and include exercising more, so the process that we just went through certainly brought that and emphasized how important that is, especially when I know how it's affecting my feet and my legs, and being a diabetic, you have to keep your circulation going. It's caused me to think about and reflect on what I need to do to relax more and rest more, and just be at a point where I don't have to do anything if I don't feel like it." (P8)

Similarly, P6 described a change she would make to address the chronic pain in her joints and back. The practice of avoiding the stairs to reduce pain conflicted with the physical activity she needed to manage diabetes and arthritis. The study activities prompted P6 to think back and come to the realization that she had kept up certain diet and exercise routines for many years out of habit rather than intention:

"It seems like I'm just-how do I put it? It's like I'm so accustomed to doing things a certain way, I keep doing it that way, not changing. Just like I'm just not thinking about what I'm doing, just doing it. Some habits need to be changed." (P6)

P10 had been considering retirement and found that the study activities helped her to clarify her priorities as she weighed her decision about retirement. Between the first and second home visits, she kept the index cards with her and carried them to her job as a bus driver. P10 explained that she thought about the cards and what she had shared with the interviewers during her breaks between routes, especially what she meant by her personal value of Freedom. Although she loved her job as a bus driver, P10 had been considering retirement so that she could fulfill her dream of experiencing Freedom while living on her own in a motor home. P10 found having the index cards with her to be useful for elucidating her motivations for retirement:

"It basically brought to light what's important to me, what's really important and what I really like and it actually helped me make decisions as to what I'm going to do. Because I was like in a total-I was torn, I don't know what to do or which way to go. I am going to retire and I'm going to enjoy the rest of my life, doing whatever I want to do, that's what I'm going to do. I need that freedom and I might just disappear for a little while. I need that." (P10)

P10 found reflection on her personal values and self-care to be useful for considering a major life change. In describing how she came to this decision, P10 explained that she did not worry about her ability to maintain her daily self-care routines and had used the cards to plan for how she would make adjustments to self-care while on the road.

Experiencing little to no change. We found that there were varying degrees to which participants found the study activities useful and benefited from thinking about their values and self-care in new ways. Patients who experienced little to no changes in how they thought about their values or self-care described that they did not discover or learn anything as a result of completing study activities. For example, 
P3 expressed that the discussion covered things he already thought about:

\begin{abstract}
“There wasn't anything cathartic about it. I've been thinking of all this for many a year now." (P3)
\end{abstract}

\section{DISCUSSION}

In this section we discuss how our findings extend prior work in HCI on designing to support reflection. The themes in our findings closely resemble levels or dimensions of reflection described in prior literature. We build on this work by demonstrating that reflection is a critical first step to articulating one's values. Our findings have implications on the design of future tools that may help improve chronic illness care by encouraging patients to reflect on values and self-care.

To clarify the contribution of this study, we draw connections between our findings and prior work on supporting reflection. First, our themes align closely with Fleck and Fitzpatrick's [20] levels of reflection, starting from the lowest to highest: Description, Reflective description, Dialogic reflection, Transformative reflection, and Critical reflection. For example, our themes of Raising awareness, evolving perspectives, and recognizing misalignments resemble Dialogic reflection, which refers to "A different level of thinking about. Looking for relationships between pieces of experience or knowledge, evidence of cycles of interpreting and questioning, consideration of different explanations, hypothesis and other points of view"[20]. Our theme of Considering changes aligns with Transformative reflection.

Additionally, we find synergies between our descriptive themes and Baumer's dimensions of reflection: Breakdown (i.e.,"situations of surprise, uncertainty, or conflict"), Inquiry (i.e., "process of revisiting concepts, ideas, theories, etc., already learned"), and Transformation (i.e, involving change or the altering of a situation) [4]. We build upon this prior literature by bringing a focus on values, beyond personal informatics data. Further, we offer a context for approaching the activity of reflection as inherently beneficial rather than as a means of persuasion toward making individual behavior changes. Reflection may be an important aspect of helping people with MCC address the overwhelming burden of treatment they frequently experience. This is of particular importance in the context of MCC because patients often make decisions about self-care priorities on their own rather than consulting with healthcare providers [36]. Our findings may help inform future work on designing to help address the burden of treatment for people with MCC.

\section{Design implications}

Our findings have a number of implications for the design of tools to support patients in helping to surface the complex relationships between personal values and the demands of self-care.

First, our findings demonstrate that self-reflection is a critical step for helping patients articulate their values and priorities. In our study, values were not pre-defined and articulating values required some reflection over time. P9 took several days to reflect and ensure he was being truthful to himself. Thus, reflection tools should allow for sufficient time for documenting values. After the first interview, P10 carried her index cards with her so that she could examine and think about what she had written during her breaks at work as a bus driver. This suggests that the portability of tools may be important to allow for reflection during convenient times and locations in daily life. Structured activities (e.g., written worksheet) for discovery and exploration may be useful for reflection in advance of eliciting values. Displaying all values to review at a glance can also promote reflection, as we discovered when seeing values cards together helped raise consciousness. Further, providing opportunities to modify values allows for patients' perspectives to evolve. While prior work on values elicitation tools for medical decisionmaking are closed-ended by design [44], tools to support reflection on values should provide ample opportunities to make modifications after initial elicitation. Major changes in life or health (e.g., retirement, death of a spouse) may be opportune moments for these tools to prompt people with MCC, most of whom are older adults, to revisit and reflect further on how their values and self-care relate.

Second, values and self-care may consist of routine, everyday practices, yet thinking about them in new ways (e.g., assigning ratings of importance or spatially arranging cards to describe connections) facilitated reflection among participants. Digital technologies may be especially helpful for creating such occasions to "defamiliarize" [8], or challenge people to think about their well-being and health in alternative ways. Designing opportunities to challenge assumptions about routinized practices that may be potentially harmful may allow for deep reflection on motivations for self-care. Also, tools for reflection might help people keep track of how their perspectives evolve or any discoveries they make about misalignments or desired changes.

Building on prior work on external representations important for sensemaking [23] and visualizations or physical constructions of personal data enable reflection [38], our findings suggest that techniques encouraging externalization can aid reflection on values and self-care. Interestingly, prompting participants to simply describing their personal values in writing or speech encouraged reflection because 
they did not routinely articulate their values. This finding challenges the assumption that personal values are already known and understood and simply need to be elicited. Our work indicates that the extra work of externalizing one's values can help people with MCC bring awareness to and make new discoveries about their daily routines.

Third, most participants responded positively to reflection guided by the interviewers. This suggests that some aspects of reflection may be best supported by human facilitators who actively listen and acknowledge a patient's values rather than through a digital interface. This offers a different take on reflection compared to prior work, much of which presents users with visualizations designed to help them progress toward a predetermined health goal.

Our finding that activities for self-reflection produced varied responses indicates that personalized techniques for reflection may produce better results. Not all patients will find reflection useful. The benefits of reflective activities may also vary depending on other aspects of a patient's life and whether they are experiencing changes in health or life. For example, P10 was already considering retirement before deciding to participate in the study, so the study activities were especially impactful because she had already been weighing this major life decision. Assessing readiness for reflection may facilitate a more meaningful reflection experience.

Finally, despite prior work demonstrating the important role of family caregivers (CGs) in chronic illness care, our results show that CGs may hinder self-reflection. This finding contradicts findings from prior work on the role of the caregiver in co-constructing values and performing self-care work [11]. Our findings that CGs interfered or disagreed with patients' reflective processes implies that patients may benefit more from reflection as an individual activity without involvement of others. However, this also raises a need for future work to consider accessibility concerns for those who rely on CGS after experiencing loss in vision or fine motor skills due to common complications of diabetes.

\section{Opportunities for future work}

Our analysis focused on the techniques that contributed to reflection among patients. Further analysis is needed to understand the types of connections patients articulated between their values and self-care, and how to explicitly encourage reflection on these connections.

Finally, these findings are limited to people receiving care in the same integrated healthcare system and in stable housing situations. There is an opportunity to better understand how the same techniques may lead to different responses in people of varying socioeconomic backgrounds, in different housing situations, or with different access to healthcare.

\section{CONCLUSION}

In order to improve healthcare for MCC, there is a need to support patients and providers in aligning their health care priorites. One barrier to this alignment is that patients often do not share what is important for their well-being and health (i.e., personal values) with healthcare providers, particularly when they do not see how their values connect with the work they do to manage their health (self-care). In this study, we identified techniques to help patients reflect on and articulate relationships between personal values and selfcare. Our findings extend recent research in HCI that sought to support personal reflection through the design of interactive technologies. We demonstrate reflection techniques that can bring one new awareness and understanding regarding personal values, self-care, and relationships between them.

\section{ACKNOWLEDGMENTS}

The authors would like to thank Evette Ludman and Christopher Mack for their valuable contributions to this study.

Research reported in this publication was supported by the National Library Of Medicine of the National Institutes of Health under Award Number R01LM012813. The content is solely the responsibility of the authors and does not necessarily represent the official views of the National Institutes of Health.

\section{REFERENCES}

[1] Rikke Aarhus and Stinne Aaløkke Ballegaard. 2010. Negotiating Boundaries: Managing Disease at Home. In Proceedings of the SIGCHI Conference on Human Factors in Computing Systems (CHI '10). ACM, New York, NY, USA, 1223-1232. https://doi.org/10.1145/1753326.1753509

[2] American Geriatrics Society Expert Panel on the Care of Older Adults with Multimorbidity. 2012. Guiding Principles for the Care of Older Adults with Multimorbidity: An Approach for Clinicians. Journal of the American Geriatrics Society 60, 10 (Oct. 2012), 1957-1968. https: //doi.org/10.1111/j.1532-5415.2012.04187.x

[3] Jessica S Ancker, Holly O Witteman, Baria Hafeez, Thierry Provencher, Mary Van de Graaf, and Esther Wei. 2015. "You Get Reminded You're a Sick Person": Personal Data Tracking and Patients With Multiple Chronic Conditions. Journal of Medical Internet Research 17, 8 (Aug. 2015), e202. https://doi.org/10.2196/jmir.4209

[4] Eric P.S. Baumer. 2015. Reflective Informatics: Conceptual Dimensions for Designing Technologies of Reflection. In Proceedings of the 33rd Annual ACM Conference on Human Factors in Computing Systems - CHI '15. ACM Press, Seoul, Republic of Korea, 585-594. https://doi.org/10. 1145/2702123.2702234

[5] Eric P.S. Baumer, Vera Khovanskaya, Mark Matthews, Lindsay Reynolds, Victoria Schwanda Sosik, and Geri Gay. 2014. Reviewing reflection: on the use of reflection in interactive system design. In Proceedings of the 2014 conference on Designing interactive systems - DIS '14. ACM Press, Vancouver, BC, Canada, 93-102. https: //doi.org/10.1145/2598510.2598598

[6] Elizabeth A. Bayliss, Denise E. Bonds, Cynthia M. Boyd, Melinda M. Davis, Bruce Finke, Michael H. Fox, Russell E. Glasgow, Richard A. Goodman, Suzanne Heurtin-Roberts, Sue Lachenmayr, Cristin Lind, Elizabeth A. Madigan, David S. Meyers, Suzanne Mintz, Wendy J. 
Nilsen, Sally Okun, Sarah Ruiz, Marcel E. Salive, and Kurt C. Stange 2014. Understanding the Context of Health for Persons With Multiple Chronic Conditions: Moving From What Is the Matter to What Matters. The Annals of Family Medicine 12, 3 (May 2014), 260-269. https: //doi.org/10.1370/afm.1643

[7] Elizabeth A. Bayliss, John F. Steiner, Douglas H. Fernald, Lori A. Crane, and Deborah S. Main. 2003. Descriptions of Barriers to Self-Care by Persons with Comorbid Chronic Diseases. The Annals of Family Medicine 1, 1 (May 2003), 15-21. https://doi.org/10.1370/afm.4

[8] Genevieve Bell, Mark Blythe, and Phoebe Sengers. 2005. Making by making strange: Defamiliarization and the design of domestic technologies. ACM Transactions on Computer-Human Interaction 12, 2 (June 2005), 149-173. https://doi.org/10.1145/1067860.1067862

[9] Andrew B.L. Berry, Catherine Lim, Andrea L. Hartzler, Tad Hirsch, Evette Ludman, Edward H. Wagner, and James D. Ralston. 2017. Creating Conditions for Patients' Values to Emerge in Clinical Conversations: Perspectives of Health Care Team Members. In Proceedings of the 2017 Conference on Designing Interactive Systems. ACM, Edinburgh, United Kingdom, 1165-1174. https://doi.org/10.1145/3064663.3064669

[10] Andrew B.L. Berry, Catherine Lim, Andrea L. Hartzler, Tad Hirsch, Evette J. Ludman, and Edward H. Wagner. 2017. Eliciting Values of Patients with Multiple Chronic Conditions: Evaluation of a Patientcentered Framework. AMIA Annual Symposium Proceedings 2017 (2017), 430.

[11] Andrew B.L. Berry, Catherine Lim, Andrea L. Hartzler, Tad Hirsch, Edward H. Wagner, Evette Ludman, and James D. Ralston. 2017. How Values Shape Collaboration Between Patients with Multiple Chronic Conditions and Spousal Caregivers. In Proceedings of the 2017 CHI Conference on Human Factors in Computing Systems (CHI '17). ACM, Denver, CO, 5257-5270. https://doi.org/10.1145/3025453.3025923

[12] Andrew B.L. Berry, Catherine Y. Lim, Andrea L. Hartzler, Tad Hirsch, Evette Ludman, Edward H. Wagner, and James D. Ralston. 2017. "It's good to know you're not a stranger every time": Communication about Values Between Patients with Multiple Chronic Conditions and Healthcare Providers. Proceedings of the ACM on Human-Computer Interaction 1, CSCW (Dec. 2017), 1-20. https://doi.org/10.1145/3134658

[13] Gabrielle S. Cantor. 2018. Designing Technological Interventions for Patients with Discordant Chronic Comorbidities and Type-2 Diabetes. In Extended Abstracts of the 2018 CHI Conference on Human Factors in Computing Systems - CHI '18. ACM Press, Montreal QC, Canada, 1-6. https://doi.org/10.1145/3170427.3180304

[14] Yunan Chen. 2010. Take it personally: accounting for individual difference in designing diabetes management systems. In Proceedings of the 8th ACM Conference on Designing Interactive Systems - DIS '10. ACM Press, Aarhus, Denmark, 252. https://doi.org/10.1145/1858171.1858218

[15] Eun Kyoung Choe, Bongshin Lee, Haining Zhu, Nathalie Henry Riche, and Dominikus Baur. 2017. Understanding self-reflection: how people reflect on personal data through visual data exploration. In Proceedings of the 11th EAI International Conference on Pervasive Computing Technologies for Healthcare - PervasiveHealth '17. ACM Press, Barcelona, Spain, 173-182. https://doi.org/10.1145/3154862.3154881

[16] Juliet Corbin and Anselm Strauss. 1985. Managing chronic illness at home: Three lines of work. Qualitative Sociology 8, 3 (1985), 224-247. https://doi.org/10.1007/BF00989485

[17] Juliet M. Corbin and Anselm Strauss. 1988. Unending work and care: Managing chronic illness at home. Jossey-Bass, San Francisco, California, USA.

[18] Emanuel EJ and Emanuel LL. 1992. Four models of the physicianpatient relationship. FAMA 267, 16 (April 1992), 2221-2226. https: //doi.org/10.1001/jama.1992.03480160079038

[19] Jordan Eschler, Logan Kendall, Kathleen O'Leary, Lisa M. Vizer, Paula Lozano, Jennifer B. McClure, Wanda Pratt, and James D. Ralston. 2015.
Shared Calendars for Home Health Management. In Proceedings of the 18th ACM Conference on Computer Supported Cooperative Work \& Social Computing - CSCW '15. ACM Press, Vancouver, BC, Canada, 1277-1288. https://doi.org/10.1145/2675133.2675168

[20] Rowanne Fleck and Geraldine Fitzpatrick. 2010. Reflecting on reflection: framing a design landscape. In Proceedings of the 22nd Conference of the Computer-Human Interaction Special Interest Group of Australia on Computer-Human Interaction - OZCHI '10. ACM Press, Brisbane, Australia, 216. https://doi.org/10.1145/1952222.1952269

[21] Martin Fortin, Gina Bravo, Catherine Hudon, Alain Vanasse, and Lise Lapointe. 2005. Prevalence of Multimorbidity Among Adults Seen in Family Practice. The Annals of Family Medicine 3, 3 (May 2005), 223-228. https://doi.org/10.1370/afm.272

[22] Erik Grönvall and Nervo Verdezoto. 2013. Beyond Self-monitoring: Understanding Non-functional Aspects of Home-based Healthcare Technology. In Proceedings of the ACM International foint Conference on Pervasive and Ubiquitous Computing. ACM, Zurich, Switzerland, 587-596. https://doi.org/10.1145/2493432.2493495

[23] David Kirsh. 2010. Thinking with external representations. AI \& SOCIETY 25, 4 (Nov. 2010), 441-454. https://doi.org/10.1007/ s00146-010-0272-8

[24] Christopher A. Le Dantec and W. Keith Edwards. 2008. Designs on dignity: perceptions of technology among the homeless. In Proceeding of the twenty-sixth annual CHI conference on Human factors in computing systems - CHI '08. ACM Press, Florence, Italy, 627. https://doi.org/10.1145/1357054.1357155

[25] Ian Li, Anind Dey, and Jodi Forlizzi. 2010. A stage-based model of personal informatics systems. In Proceedings of the 28th international conference on Human factors in computing systems - CHI '10. ACM Press, Atlanta, Georgia, USA, 557. https://doi.org/10.1145/1753326.1753409

[26] Catherine Lim, Andrew B.L. Berry, Tad Hirsch, Andrea L. Hartzler, Edward H. Wagner, Evette Ludman, and James D. Ralston. 2016. "It Just Seems Outside My Health": How Patients with Chronic Conditions Perceive Communication Boundaries with Providers. In Proceedings of the 2016 ACM Conference on Designing Interactive Systems (DIS '16). ACM, Brisbane, Australia, 1172-1184. https://doi.org/10.1145/2901790. 2901866

[27] Catherine Y. Lim, Andrew B. L. Berry, Tad Hirsch, Andrea L. Hartzler, Edward H. Wagner, Evette J. Ludman, and James D. Ralston. 2017. Understanding What Is Most Important to Individuals with Multiple Chronic Conditions: A Qualitative Study of PatientsâĂŹ Perspectives. fournal of General Internal Medicine 32, 12 (Dec. 2017), 1278-1284. https://doi.org/10.1007/s11606-017-4154-3

[28] Kate R. Lorig and Halsted R. Holman. 2003. Self-management education: History, definition, outcomes, and mechanisms. Annals of Behavioral Medicine 26, 1 (Aug. 2003), 1-7. https://doi.org/10.1207/ S15324796ABM2601 01

[29] Lena Mamykina, Elizabeth Mynatt, Patricia Davidson, and Daniel Greenblatt. 2008. MAHI: investigation of social scaffolding for reflective thinking in diabetes management. In Proceeding of the twenty-sixth annual CHI conference on Human factors in computing systems - CHI '08. ACM, Florence, Italy, 477. https://doi.org/10.1145/1357054.1357131

[30] Lena Mamykina, Arlene M. Smaldone, and Suzanne R. Bakken. 2015. Adopting the sensemaking perspective for chronic disease selfmanagement. fournal of Biomedical Informatics 56 (Aug. 2015), 406-417. https://doi.org/10.1016/j.jbi.2015.06.006

[31] David Markland, Richard M. Ryan, Vannessa Jayne Tobin, and Stephen Rollnick. 2005. Motivational Interviewing and Self-Determination Theory. Journal of Social and Clinical Psychology 24, 6 (Sept. 2005), 811-831. https://doi.org/10.1521/jscp.2005.24.6.811

[32] Aanand D. Naik, Lindsey A. Martin, Jennifer Moye, and Michele J. Karel. 2016. Health Values and Treatment Goals of Older, Multimorbid Adults 
Facing Life-Threatening Illness. Journal of the American Geriatrics Society 64, 3 (March 2016), 625-631. https://doi.org/10.1111/jgs.14027

[33] Naik AD. 2013. On the road to patient centeredness: Comment on: "communication and medication refill adherence". FAMA Internal Medicine 173, 3 (Feb. 2013), 218-219. https://doi.org/10.1001/ jamainternmed.2013.1229

[34] Francisco Nunes and Geraldine Fitzpatrick. 2015. Self-Care Technologies and Collaboration. International Journal of Human-Computer Interaction 31, 12 (Dec. 2015), 869-881. https://doi.org/10.1080/10447318. 2015.1067498

[35] Leysia Palen and Stinne Aaløkke. 2006. Of pill boxes and piano benches: "home-made" methods for managing medication. In Proceedings of the 2006 20th anniversary conference on Computer supported cooperative work. ACM, Banff, Alberta, Canada, 79-88. https://doi.org/10.1145/ 1180875.1180888

[36] Michael Rosbach and John Sahl Andersen. 2017. Patient-experienced burden of treatment in patients with multimorbidity: A systematic review of qualitative data. PLOS ONE 12, 6 (June 2017), e0179916. https://doi.org/10.1371/journal.pone.0179916

[37] Herman Saksono and Andrea G. Parker. 2017. Reflective Informatics Through Family Storytelling: Self-discovering Physical Activity Predictors. In Proceedings of the 2017 CHI Conference on Human Factors in Computing Systems - CHI '17. ACM Press, Denver, Colorado, USA, 5232-5244. https://doi.org/10.1145/3025453.3025651

[38] Alice Thudt, Uta Hinrichs, Samuel Huron, and Sheelagh Carpendale. 2018. Self-Reflection and Personal Physicalization Construction. In Proceedings of the 2018 CHI Conference on Human Factors in Computing Systems - CHI '18. ACM Press, Montreal QC, Canada, 1-13. https: //doi.org/10.1145/3173574.3173728

[39] Katrin Uhlig, Bruce Leff, David Kent, Sydney Dy, Klara Brunnhuber, Jako S. Burgers, Sheldon Greenfield, Gordon Guyatt, Kevin High, Rosanne Leipzig, Cynthia Mulrow, Kenneth Schmader, Holger Schunemann, Louise C. Walter, James Woodcock, and Cynthia M. Boyd. 2014. A Framework for Crafting Clinical Practice Guidelines that are Relevant to the Care and Management of People with Multimorbidity. Fournal of General Internal Medicine 29, 4 (April 2014), 670-679. https://doi.org/10.1007/s11606-013-2659-y

[40] Chris Van Weel and François G Schellevis. 2006. Comorbidity and guidelines: conflicting interests. The Lancet 367, 9510 (Feb. 2006), 550-551. https://doi.org/10.1016/S0140-6736(06)68198-1

[41] Arjun Venkatesh, Kate Goodrich, and Patrick H. Conway. 2014. Opportunities for Quality Measurement to Improve the Value of Care for Patients With Multiple Chronic Conditions. Annals of Internal Medicine 161, 10_Supplement (Nov. 2014), S76. https://doi.org/10. 7326/M13-3014

[42] Isabel Voigt, Jennifer Wrede, Heike Diederichs-Egidi, Marie-Luise Dierks, and Ulrike Junius-Walker. 2010. Priority Setting in General Practice: Health Priorities of Older Patients Differ from Treatment Priorities of Their Physicians. Croatian Medical fournal 51, 6 (Dec. 2010), 483-492. https://doi.org/10.3325/cmj.2010.51.483

[43] Carlos O. Weiss, Cynthia M. Boyd, Qilu Yu, Jennifer L. Wolff, and Bruce Leff. 2007. Patterns of prevalent major chronic disease among older adults in the United States. FAMA 298, 10 (Sept. 2007), 1158-1162. https://doi.org/10.1001/jama.298.10.1160-b

[44] Holly O. Witteman, Laura D. Scherer, Teresa Gavaruzzi, Arwen H. Pieterse, Andrea Fuhrel-Forbis, Selma Chipenda Dansokho, Nicole Exe, Valerie C. Kahn, Deb Feldman-Stewart, Nananda F. Col, Alexis F. Turgeon, and Angela Fagerlin. 2016. Design Features of Explicit Values Clarification Methods: A Systematic Review. Medical Decision Making 36, 4 (May 2016), 453-471. https://doi.org/10.1177/0272989X15626397

[45] Donna M. Zulman, Steven M. Asch, Susana B. Martins, Eve A. Kerr, Brian B. Hoffman, and Mary K. Goldstein. 2014. Quality of Care for Patients with Multiple Chronic Conditions: The Role of Comorbidity Interrelatedness. Fournal of General Internal Medicine 29, 3 (March 2014), 529-537. https://doi.org/10.1007/s11606-013-2616-9

[46] Donna M. Zulman, Eve A. Kerr, Timothy P. Hofer, Michele Heisler, and Brian J. Zikmund-Fisher. 2010. Patient-provider concordance in the prioritization of health conditions among hypertensive diabetes patients. Journal of General Internal Medicine 25, 5 (2010), 408-414. 